03,08, 12

\title{
Неуниверсальность частотной зависимости проводимости неупорядоченных наногранулированных систем
}

\author{
(C) М.А. Ормонт, И.П. Звягин \\ Московский государственный университет им. М.В. Ломоносова \\ Москва, Россия \\ E-mail: ormont@phys.msu.ru
}

(Поступила в Редакцию 13 июня 2018 г.)

Для неупорядоченного массива плотно упакованных сферических наногранул проведен расчет вещественной части высокочастотной бесфононной проводимости в парном приближении. Обобщение теории бесфононной проводимости систем с точечными примесными центрами на системы с областями локализации конечных размеров (массивы наногранул или квантовых точек) показало, что на высокочастотную проводимость влияет функция распределения расстояний между поверхностями гранул $P(w)$; это может приводить к отклонениям от линейной частотной зависимости вещественной части проводимости $\sigma_{1}(\omega)$. В окрестности частоты $\omega \sim \omega_{c}=2 I_{0} / \hbar\left(I_{0}-\right.$ предэкспоненциальный множитель резонансного интеграла) для неупорядоченных гранулированных систем следует ожидать отклонений от универсальности $\sigma_{1}(\omega) \sim \omega^{s}$ $(s \approx 1)$, связанных с ослаблением частотной зависимости проводимости $\sigma_{1}(\omega)$, и ее немонотонности. С увеличением размера гранул немонотонность $\sigma_{1}(\omega)$ должна проявляться при меньших частотах; это связано с уменьшением предэкспоненциального множителя $I_{0}$ резонансного интеграла с увеличением размера гранул.

DOI: 10.21883/FTT.2018.12.46721.156

\section{1. Введение}

Как известно, исследования частотных зависимостей функций линейного отклика среды (комплексной диэлектрической восприимчивости $\alpha(\omega)$, проницаемости $\varepsilon(\omega)$ и проводимости $\sigma(\omega)$ ) может дать информацию об особенностях механизмов переноса носителей заряда и о локальной структуре материала [1].

Для многих неупорядоченных материалов (аморфные и легированные полупроводники, полупроводниковые стекла, проводящие полимеры, гранулированные материалы и т.п.) частотная зависимость проводимости в широком интервале частот (вплоть до $\mathrm{GHz}$ ) может быть описана степенной зависимостью $\sigma(\omega)=\sigma_{1}(\omega)+i \sigma_{2}(\omega) \sim \omega^{s}$, где $s-$ постоянная $(0.7<s<1)$ [2]. Возрастающая степенная частотная зависимость проводимости обычно свидетельствует о прыжковом механизме электронного переноса.

Универсальность степенной зависимости проводимости $\sigma(\omega) \sim \omega^{s}(s<1)$ затрудняет получение информации о конкретных особенностях механизма переноса. По этой причине исследования отклонений от универсальности и нахождение их связи со структурными особенностями материала и с особенностями переноса играют важную роль.

В случае прыжкового переноса по локализованным состояниям примесной зоны слабо легированных компенсированных полупроводников теория предсказывает частотные зависимости вещественной $\sigma_{1}(\omega)$ и мнимой $\sigma_{2}(\omega)$ частей комплексной проводимости вида $\sigma_{1}(\omega)$, $\sigma_{2}(\omega) \sim r_{\omega}^{n} \omega^{m} \sim \omega^{m} \ln ^{n}\left(\omega_{\mathrm{c}, \mathrm{ph}} / \omega\right)$ (где $m, n$ - целые числа, $r_{\omega}$ - характерная длина прыжка на частоте $\omega, \omega_{\mathrm{ph}}-$ характерная фононная частота, представляющая собой частоту попыток перехода электрона при релаксационной прыжковой проводимости; $\omega_{\mathrm{c}}$ - частота попыток перехода электрона при резонансной проводимости). Функции вида $\omega^{m} \ln ^{n}\left(\omega_{\mathrm{c}, \mathrm{ph}} / \omega\right)$, фигурирующие в выражениях для проводимости $\sigma_{1}(\omega)$ и $\sigma_{2}(\omega)$ при $\omega \ll \omega_{\mathrm{c}, \mathrm{ph}}$ можно аппроксимировать степенным законом $A \omega^{s}$ с показателем степени $s=\frac{d \ln (\sigma(\omega))}{d \ln \omega}=m-\frac{n}{\ln \left(\omega_{\mathrm{c}, \mathrm{ph}} / \omega\right)}$; при этом показатель степени $s$ уменьшается с ростом частоты $\omega$ [3].

Степенную частотную зависимость вещественной части проводимости с показателем степени $s \approx 1$ обычно принято связывать с прыжковой проводимостью с переменной длиной прыжка по локализованным состояниям с участием фононов (релаксационная проводимость) [4-6]. Частотная зависимость вещественной части проводимости, близкая к линейной, получается при не слишком высоких частотах и в теории низкотемпературной бесфононной (резонансной) прыжковой проводимости при учете кулоновских корреляций [7]. Теория бесфононной проводимости предсказывает переход (кроссовер) от линейной частотной зависимости $(s \approx 1)$ к зависимости, близкой к квадратичной $(s \approx 2)$ в области частот порядка $\omega_{\mathrm{cr}}$, при которых $\hbar \omega$ становится порядка энергии кулоновского взаимодействия между электронами внутри резонансных пар [7,8]; при более низких частотах вещественная часть проводимости определяется фононным механизмом, а с ростом частоты бесфононная проводимость начинает преобладать над релаксационной. Низкотемпературные (при $T \sim 1 \mathrm{~K})$ измерения частотной зависимости проводимости в легированном кремнии ( $\mathrm{Si}: \mathrm{B}[9], \mathrm{Si}: \mathrm{P}[10,11])$ 
на изоляторной стороне перехода металл-диэлектрик и в металлических нанокомпозитах [12] показали, что с ростом частоты зависимость вещественной части проводимости $\sigma_{1}(\omega)$ переходит от линейной к квадратичной. $\mathrm{B} \mathrm{Si}: \mathrm{P}$ этот переход происходит при частотах порядка $v_{\mathrm{cr}} \sim 1 \mathrm{THz}[10,11]$.

Проведенное в работе [13] рассмотрение показало, что в силу частотной зависимости характерной длины прыжка $r_{\omega}$ частотная зависимость $\sigma_{1}(\omega)$ может быть немонотонной, причем вплоть до частоты $\omega_{m}$, отвечающей максимуму $\sigma_{1}(\omega)$, кулоновское взаимодействие между электронами „активных“ (резонансных) пар играет основную роль при $\hbar \omega<e^{2} / \kappa r_{\omega}$ и частотная зависимость $\sigma_{1}(\omega)$ остается близкой к линейной. Однако предсказанная теорией немонотонность частотной зависимости бесфононной проводимости в легированных компенсированных полупроводниках экспериментально не была обнаружена $[10,11]$. Наблюдаемый переход от линейной к квадратичной частотной зависимости проводимости $\sigma_{1}(\omega)$ может быть связан с переходом к режиму проводимости с постоянной длиной прыжка, когда основной вклад в проводимость вносят электронные переходы внутри пар с оптимальными межцентровыми расстояниями $r_{\text {opt }}$, слабо зависящими от частоты [14-17]. Существование оптимального расстояния $r_{\text {opt }}$ между центрами в парах обусловлено тем, что с уменьшением расстояния между центрами в паре уменьшается и изменение дипольного момента системы при электронном переходе, а с увеличением расстояния между центрами происходит экспоненциальное уменьшение перекрытия волновых функций состояний, отвечающих центрам локализации. С ростом частоты характерная длина прыжка $r_{\omega}$ уменьшается. Переход к режиму проводимости с постоянной длиной прыжка $r_{\text {opt }}$ происходит в области частот, где $r_{\mathrm{opt}}>r_{\omega}$; в этом случае основной вклад в проводимость вносят электронные переходы внутри пар с межцентровыми расстояниями $r_{\lambda, \lambda^{\prime}} \sim r_{\text {орt }}$.

Имеющаяся теория бесфононной проводимости неупорядоченных полупроводников с мелкими примесными уровнями не учитывает неточечности центров локализации носителей заряда; в тоже время на практике часто приходится иметь дело с неупорядоченными системами, включающими в себя центры локализации конечных размеров (наногранулы, квантовые точки, проводящие области в легированных компенсированных полупроводниках, обусловленные крупномасштабными флуктуациями концентрации примеси и связанные с крупномасштабными флуктуациями случайных кулоновских полей) $[18,19]$. Отметим, что высокочастотная электронная проводимость, описываемая в рамках парного приближения, существенно зависит от особенностей структуры центров локализации; при этом особый интерес представляют аномалии прыжковых явлений переноса, связанные с взаимным влиянием квантового ограничения, беспорядка и кулоновского взаимодействия электронов. В работе [20] было показано, что учет размеров гранул существенно влияет на результат при вычислении высокочастотной релаксационной прыжковой проводимости массива плотно упакованных гранул. В частности, в режиме постоянной не зависящей от частоты длины прыжка (переходы между ближайшими соседями) высокочастотная релаксационная проводимость массива плотно упакованных гранул зависит от функции распределения $P\left(w_{\lambda \lambda^{\prime}}\right)$ величин расстояний между поверхностями гранул $w_{\lambda \lambda^{\prime}}$. Зависимость проводимости $\sigma(\omega)$ от структурной характеристики $P\left(w_{\lambda \lambda^{\prime}}\right)$ может приводить к отклонениям частотной зависимости вещественной части проводимости $\sigma_{1}(\omega)$ от линейной.

Вместе с тем, в области высоких частот бесфононная составляющая проводимости $\sigma_{1}^{\text {res }}$ преобладает над релаксационной $\sigma_{1}^{\text {rel }}$; при этом $\sigma_{1}(\omega)=\sigma_{1}^{\text {res }}(\omega)+\sigma_{1}^{\text {rel }}(\omega)$ $\approx \sigma_{1}^{\text {res }}(\omega)$. Проведем обобщение теории высокочастотной бесфононной проводимости систем с точечными примесными центрами на системы с областями локализации конечных размеров (массивы наногранул или квантовых точек).

Рассмотрим низкотемпературную бесфононную проводимость неупорядоченной системы, обусловленную электронными переходами между состояниями, отвечающими различным областям локализации конечных размеров.

\section{2. Модель гранулированной системы}

Рассмотрим систему, включающую массив сферических наногранул или квантовых точек, с беспорядком, возникающим из-за случайных флуктуаций размера гранул $d_{\lambda}$ и расстояний между поверхностями гранул $w_{\lambda, \lambda^{\prime}}$ (ширин потенциальных диэлектрических барьеров). Флуктуации размера гранул, эффекты размерного квантования, кулоновского взаимодействия, квантовой корреляции (квантового отталкивания) уровней энергии приводят к случайному разбросу энергий локализованных состояний, связанных с гранулами. Будем рассматривать случай плотной упаковки гранул, когда длина затухания $\gamma^{-1}$ волновых функций в областях диэлектрических барьеров между гранулами и среднее расстояние $w$ между поверхностями ближайших (соседних) гранул меньше среднего размера гранул $d$, т. е. $\gamma^{-1}<w<d$.

В области высоких частот, больших частоты, при которой длина туннелирования становится сравнима с диаметром гранулы, проводимость определяется переходами между ближайшими гранулами. В этом случае при расчете высокочастотной проводимости можно ограничиться учетом перекрытия волновых функций соседних гранул и не принимать во внимание перекрытие между удаленными гранулами [20-22].

Частотная зависимость характерной длины прыжка $r_{\omega}$ в режиме резонансной проводимости связана с гибридизацией электронных состояний. Согласно теории $[7,8]$ для мелких примесных уровней, из-за гибридизации волновых функций изолированной пары центров и соответствующего ей отталкивания уровней, наибольший вклад 
в бесфононную проводимость вносят пары центров, для которых межцентровые расстояния $r_{\lambda, \lambda^{\prime}}$ удовлетворяют неравенствам $r_{\omega} \leq r_{\lambda \lambda^{\prime}} \leq r_{\omega}+a$ ( $a-$ радиус локализации); при $r_{\lambda \lambda^{\prime}}<r_{\omega}$ отталкивание уровней за счет гибридизации состояний приводит к тому, что $\varepsilon_{\lambda \lambda^{\prime}}^{+}-\varepsilon_{\lambda \lambda^{\prime}}^{-}>\hbar \omega$, и резонансные переходы невозможны. В случае достаточно большого разброса энергий уровней, при условии $\left|\varepsilon_{\lambda^{\prime \prime}}^{0}-\varepsilon_{\lambda}^{0}\right| \gg\left|I_{\lambda, \lambda^{\prime \prime}}\right|$, $\left|\varepsilon_{\lambda^{\prime \prime}}^{0}-\varepsilon_{\lambda^{\prime}}^{0}\right| \gg\left|I_{\lambda^{\prime}, \lambda^{\prime \prime}}\right|$, пары центров $\lambda, \lambda^{\prime}$ с близкими энергиями $\varepsilon_{\lambda}^{0} \approx \varepsilon_{\lambda^{\prime}}^{0}$ можно считать изолированными; при учете гибридизации локализованных электронных состояний в таких парах перекрытием волновых функций центров $\lambda, \lambda^{\prime}$ с другими центрами $\lambda^{\prime \prime}$ можно пренебречь. Характерная длина прыжка $r_{\omega}$ в парном приближении на частоте $\omega$ определяется из условия $\hbar \omega=2 I_{\lambda, \lambda^{\prime}}\left(r_{\omega}\right)$, где $I_{\lambda, \lambda^{\prime}}=I_{0} \exp \left(-r_{\lambda \lambda^{\prime}} / a\right)-$ резонансный интеграл, а $\lambda$ - номер центра. Следует отметить, что характерная длина прыжка $r_{\omega}$ определяется тем, что область значений $\varepsilon_{\lambda \lambda^{\prime}}^{+}-\varepsilon_{\lambda, \lambda^{\prime}}^{-}$ограничена снизу величиной $2 I\left(r_{\lambda \lambda^{\prime}}\right)$. Ограничение существенно, когда разность затравочных энергий $\varepsilon_{\lambda^{\prime}}^{0}-\varepsilon_{\lambda}^{0}$ по абсолютной величине меньше $2 I_{\lambda, \lambda^{\prime}}$. $\mathrm{C}$ ростом частоты $\omega$ характерная длина прыжка $r_{\omega}$ уменьшается.

Как и для точечных примесных центров, в случае наногранулированной системы вследствие большого разброса энергий уровней, гибридизацию волновых функций локализованных электронных состояний при расчете высокочастотной проводимости можно учесть в парном приближении, считая пару центров локализации (гранул) $\lambda, \lambda^{\prime}$ изолированной, т.е. пренебречь перекрытием волновых функций с другими центрами, не принадлежащими рассматриваемой паре.

В слабых электрических полях в парном приближении выражение для бесфононной проводимости имеет вид [23]

$$
\begin{aligned}
& \sigma_{1}^{\mathrm{res}}(\omega)=\frac{\pi e^{2} \omega}{V_{0}} \sum_{\substack{\left\{\lambda, \lambda^{\prime}\right\} \\
\lambda \neq \lambda^{\prime}}}\left|\left\langle\psi_{\lambda, \lambda^{\prime}}^{-}|(\mathbf{n}, \mathbf{r})| \psi_{\lambda, \lambda^{\prime}}^{+}\right\rangle\right|^{2} \\
& \quad \times\left(n_{F}\left(\varepsilon_{\lambda, \lambda^{\prime}}^{-}\right)-n_{F}\left(\varepsilon_{\lambda, \lambda^{\prime}}^{+}\right)\right) \delta\left(\varepsilon_{\lambda, \lambda^{\prime}}^{-}-\varepsilon_{\lambda, \lambda^{\prime}}^{+}+\hbar \omega\right),
\end{aligned}
$$

где $\psi_{\lambda, \lambda^{\prime}}^{ \pm}=C_{\lambda}^{ \pm} \psi_{\lambda}+C_{\lambda^{\prime}}^{ \pm} \psi_{\lambda^{\prime}}-$ гибридизованные волновые функции основных состояний $\psi_{\lambda}, \psi_{\lambda^{\prime}}$ электрона на изолированных центрах локализации (наногранулах) $\lambda$ и $\lambda^{\prime}, \mathbf{n}-$ единичный вектор, параллельный внешнему электрическому полю, $n_{F}(\varepsilon)-$ среднее число заполнения состояния с энергией $\varepsilon, V_{0}$ - объем системы. Волновые функции $\psi_{\lambda, \lambda^{\prime}}^{-}, \psi_{\lambda, \lambda^{\prime}}^{+}$отвечают нижнему $\varepsilon_{\lambda, \lambda^{\prime}}^{-}$ и верхнему $\varepsilon_{\lambda, \lambda^{\prime}}^{+}$уровням энергии,

$$
\varepsilon_{\lambda, \lambda^{\prime}}^{ \pm}=\frac{\varepsilon_{\lambda}^{0}+\varepsilon_{\lambda^{\prime}}^{0}}{2} \pm \frac{1}{2} \sqrt{\left(\varepsilon_{\lambda}^{0}-\varepsilon_{\lambda^{\prime}}^{0}\right)^{2}+4 I_{\lambda, \lambda^{\prime}}^{2}}
$$

$I_{\lambda, \lambda^{\prime}}=\left\langle\psi_{\lambda}\left|\hat{U}_{\lambda}\right| \psi_{\lambda^{\prime}}\right\rangle-$ резонансный интеграл; $\hat{U}_{\lambda} \approx \hat{\tilde{U}}_{\lambda}+$ $+e \varphi\left(\mathbf{r}_{\lambda}\right)$ - потенциальная энергия локализованного электрона, создаваемая центром $\lambda$ и учитывающая кулоновский сдвиг е $\varphi\left(\mathbf{r}_{\lambda}\right)$, обусловленный другими заряженными центрами в области расположения наногранулы с номером $\lambda$. При этом волновая функция локализованного в области гранулы основного состояния остается практически неизменной и близкой к волновой функции, соответствующей изолированной грануле. Волновые функции $\psi_{\lambda, \lambda^{\prime}}^{-}, \psi_{\lambda, \lambda^{\prime}}^{+}$представляют собой гибридизованные волновые функции основных состояний электрона на изолированных центрах локализации (наногранулах).

Для волновых функций основных состояний сферически симметричных гранул матричный элемент равен

$$
\begin{aligned}
\left\langle\psi_{\lambda, \lambda^{\prime}}^{-}|(\mathbf{n}, \mathbf{r})| \psi_{\lambda, \lambda^{\prime}}^{+}\right\rangle= & \left(\mathbf{n}, \mathbf{r}_{\lambda \lambda^{\prime}}\right) \frac{I_{\lambda, \lambda^{\prime}}}{\Gamma_{\lambda, \lambda^{\prime}}} \\
& +\frac{\left(\varepsilon_{\lambda}^{0}-\varepsilon_{\lambda^{\prime}}^{0}\right)\left\langle\psi_{\lambda}|(\mathbf{n}, \mathbf{r})| \psi_{\lambda^{\prime}}\right\rangle}{\Gamma_{\lambda, \lambda^{\prime}}}
\end{aligned}
$$

здесь $\left\langle\psi_{\lambda}|\mathbf{r}| \psi_{\lambda}\right\rangle=\mathbf{r}_{\lambda},\left\langle\psi_{\lambda^{\prime}}|\mathbf{r}| \psi_{\lambda^{\prime}}\right\rangle=\mathbf{r}_{\lambda^{\prime}}=\mathbf{r}_{\lambda}+\mathbf{r}_{\lambda \lambda^{\prime}}, r_{\lambda}$ радиус-вектор центра гранулы (шара) $\lambda, \quad \mathbf{r}_{\lambda \lambda^{\prime}}-$ радиус-вектор центра гранулы $\lambda^{\prime}$ относительно центра гранулы $\lambda,\left\langle\psi_{\lambda}|(\mathbf{n}, \mathbf{r})| \psi_{\lambda^{\prime}}\right\rangle=\left\langle\psi_{\lambda^{\prime}}|(\mathbf{n}, \mathbf{r})| \psi_{\lambda}\right\rangle, \quad \Gamma_{\lambda, \lambda^{\prime}}=$ $=\sqrt{\left(\varepsilon_{\lambda}^{0}-\varepsilon_{\lambda^{\prime}}^{0}\right)^{2}+4 I_{\lambda, \lambda^{\prime}}^{2}}$.

Для резонансных пар уровней с затравочными энергиями $\varepsilon_{\lambda}^{0} \approx \varepsilon_{\lambda^{\prime}}^{0}$, вносящих основной вклад в бесфононную проводимость, матричный элемент (2) принимает вид

$$
\left\langle\psi_{\lambda, \lambda^{\prime}}^{-}|(\mathbf{n}, \mathbf{r})| \psi_{\lambda, \lambda^{\prime}}^{+}\right\rangle \approx\left(\mathbf{n}, \mathbf{r}_{\lambda, \lambda^{\prime}}\right) \frac{I_{\lambda, \lambda^{\prime}}}{\Gamma_{\lambda, \lambda^{\prime}}}
$$

Отметим, что выражение для матричного элемента (2) в случае сферически симметричных гранул совпадает с выражением для матричного элемента в случае водородоподобных примесных центров (мелких локальных уровней). Согласно (3), выражение для вещественной части бесфононной проводимости неупорядоченного массива наногранул принимает вид

$$
\begin{aligned}
& \sigma_{1}^{\mathrm{res}}(\omega)=\frac{\pi e^{2} \omega}{V_{0}} \sum_{\substack{\left\{\lambda, \lambda^{\prime}\right\} \\
\lambda \neq \lambda^{\prime}}}\left[\frac{\left(\mathbf{n}, \mathbf{r}_{\lambda \lambda^{\prime}}\right) I_{\lambda, \lambda^{\prime}}}{\Gamma_{\lambda, \lambda^{\prime}}}\right]^{2} \\
& \quad \times\left(n_{F}\left(\varepsilon_{\lambda, \lambda^{\prime}}^{-}\right)-n_{F}\left(\varepsilon_{\lambda, \lambda^{\prime}}^{+}\right)\right) \delta\left(\varepsilon_{\lambda, \lambda^{\prime}}^{-}-\varepsilon_{\lambda, \lambda^{\prime}}^{+}+\hbar \omega\right) .
\end{aligned}
$$

Из теории бесфононной проводимости систем водородоподобных (точечных) центров локализации следует, что предэкспоненциальный множитель $I_{0}$ резонансного интеграла $I_{\lambda, \lambda^{\prime}}=I_{0} \exp \left(-r_{\lambda, \lambda^{\prime}} / a\right)$ служит основным параметром рассматриваемой задачи о высокочастотной проводимости, определяющим величину критической частоты $\omega_{c}=2 I_{0} / \hbar$, при которой длина прыжка становится порядка радиуса локализации состояний. Для систем с водородоподобными примесными центрами (Si : P) характерное значение критической частоты составляет $v_{c}=\omega_{c} / 2 \pi \sim 10 \mathrm{THz}$. 


\section{3. Резонансный интеграл}

В случае плотной упаковки гранул, $\gamma^{-1}<w<d$, предэкспоненциальный множитель $I_{0}$ в выражении для резонансного интеграла $I_{\lambda, \lambda^{\prime}}$ существенно уменьшается с увеличением размеров центров локализации. Этот эффект определяется двумя тенденциями. С одной стороны, в связи с условием нормировки с увеличением размера гранул происходит уменьшение амплитуд соответствующих волновых функций состояний на границах гранул; с другой, - увеличение размера гранул обусловливает увеличение объема эффективной контактной области (сферического сегмента с высотой $\gamma^{-1}$, интегрирование по которому и дает основной вклад в резонансный интеграл) [20].

Вычислим резонансный интеграл в рассматриваемом нами случае сферических наногранул. Для оценки предэкспоненциального множителя $I_{0}$ резонансного интеграла рассмотрим случай двух одинаковых сферически симметричных прямоугольных потенциальных квантовых ям радиуса $R$, для которых зависимость потенциальной энергии от расстояния до центра квантовой ямы имеет вид $\tilde{U}_{\lambda}(r)=-U_{0}$ при $r \leq R, \tilde{U}_{\lambda}(r)=0$ при $r>R$.

Как известно, волновая функция, отвечающая основному состоянию изолированной квантовой ямы, в областях внутри и вне квантовой ямы, имеет вид: $\psi_{\lambda}=A \sin (\alpha r) / r \quad$ при $\quad r \leq R, \quad \psi_{\lambda}=B \exp (-\gamma r) / r \quad$ при $r>R$; здесь $\alpha=\sqrt{2 m\left(U_{0}-\varepsilon\right)} / \hbar, \gamma=\sqrt{2 m \varepsilon} / \hbar-$ обратный радиус локализации волновой функции в области барьера между ямами, $\alpha^{2}+\gamma^{2}=2 m U_{0} / \hbar^{2}, \varepsilon=-E, E-$ энергия электрона, $m$ - эффективная масса электрона (см., например, [24]).

В случае сильной локализации волновых функций в областях квантовых ям (пренебрегая вероятностью нахождения электрона во внешней, по отношению к квантовой яме, области $r>R$ ) из условия нормировки имеем

$$
A \approx \frac{1}{\sqrt{2 \pi R\left(1-\frac{\sin (2 \alpha R)}{2 \alpha R}\right)}}
$$

при этом из непрерывности волновых функций на границе ям следует равенство $B=A \sin (\alpha R) \exp (\gamma R)$.

Отметим, что резонансный интеграл можно представить в виде

$$
I_{\lambda, \lambda^{\prime}}=\left\langle\psi_{\lambda}\left|\hat{U}_{\lambda}\right| \psi_{\lambda^{\prime}}\right\rangle \approx\left\langle\psi_{\lambda}\left|\tilde{\tilde{U}}_{\lambda}\right| \psi_{\lambda^{\prime}}\right\rangle+e \varphi\left(\mathbf{r}_{\lambda}\right) s_{\lambda, \lambda^{\prime}}
$$

здесь $\hat{U}_{\lambda} \approx \hat{\tilde{U}}_{\lambda}+e \varphi\left(\mathbf{r}_{\lambda}\right)-$ потенциальная энергия локализованного электрона, создаваемая центром $\lambda$ и учитывающая кулоновский сдвиг $е \varphi\left(\mathbf{r}_{\lambda}\right)$, обусловленный другими заряженными центрами в области расположения наногранулы с номером $\lambda$.

При решении задачи о гибридизации состояний часто можно считать, что резонансные интегралы не зависят от случайных кулоновских полей $\varphi\left(\mathbf{r}_{\lambda}\right)$, т. е.

$$
I_{\lambda, \lambda^{\prime}}=\left\langle\psi_{\lambda}\left|\hat{U}_{\lambda}\right| \psi_{\lambda^{\prime}}\right\rangle \approx\left\langle\psi_{\lambda}\left|\hat{\tilde{U}}_{\lambda}\right| \psi_{\lambda^{\prime}}\right\rangle
$$

равенства (7) справедливы при условии

$$
\left|\left\langle\psi_{\lambda}\left|\hat{\tilde{U}}_{\lambda}\right| \psi_{\lambda^{\prime}}\right\rangle\right|>\left|e \varphi\left(\mathbf{r}_{\lambda}\right) s_{\lambda, \lambda^{\prime}}\right| \text {. }
$$

Неравенство (8) представляет собой условие на глубину квантовых ям, отвечающих приближению (7). Действительно, в случае плотной упаковки гранул, $\gamma^{-1}<w_{\lambda, \lambda^{\prime}}<d=2 R$, существенный вклад в интеграл неортогональности $s_{\lambda, \lambda^{\prime}}$ дает интегрирование по межгранульной части сигарообразной области, вытянутой вдоль отрезка $r_{\lambda, \lambda^{\prime}}$, внутри межгранульной части которой произведение волновых функций $\psi_{\lambda} \psi_{\lambda^{\prime}}$ можно считать постоянным и равным

$$
\begin{aligned}
\psi_{\lambda} \psi_{\lambda^{\prime}} & \approx\left(\frac{B}{R}\right)^{2} \exp \left(-\gamma r_{\lambda, \lambda^{\prime}}\right) \\
& =\left(\frac{A \sin (\alpha R)}{R}\right)^{2} \exp \left(-\gamma w_{\lambda, \lambda^{\prime}}\right)
\end{aligned}
$$

здесь $w_{\lambda, \lambda^{\prime}}-$ расстояние между поверхностями гранул, $r_{\lambda, \lambda^{\prime}}=2 R+w_{\lambda, \lambda^{\prime}}-$ расстояние между центрами сферических гранул $\lambda$ и $\lambda^{\prime}$.

Граница сигарообразной области, интегрирование по межгранульной части которой дает существенный вклад в интеграл неортогональности $s_{\lambda, \lambda^{\prime}}$, представляет собой поверхность, на которой значение произведения

$$
\begin{aligned}
\psi_{\lambda} \psi_{\lambda^{\prime}} & \approx\left(\frac{A \sin (\alpha R)}{R}\right)^{2} \exp \left(-\gamma w_{\lambda, \lambda^{\prime}}\right) \\
& \times \exp \left(-\frac{1}{2} \frac{\gamma r_{\perp}^{2} r_{\lambda, \lambda^{\prime}}}{\left(r_{\lambda, \lambda^{\prime}}-r_{\|}\right) r_{\|}}\right)
\end{aligned}
$$

уменьшается в $e$ раз по сравнению со значением (9); площадь поперечного сечения интересующей области равна $S(\xi) \approx \pi r_{\perp}^{2} \approx \pi 2 \gamma^{-1} r_{\lambda, \lambda^{\prime}} \xi(1-\xi)$, где $\xi=r_{\|} / r_{\lambda, \lambda^{\prime}}, \xi \in\left[\frac{R}{2 R+w}, \frac{R+w}{2 R+w}\right], \mathbf{r}_{\perp}, \mathbf{r}_{\|}$- перпендикулярная и параллельная отрезку $r_{\lambda, \lambda^{\prime}}$ составляющие вектора $\mathbf{r}=\mathbf{r}_{\perp}+\mathbf{r}_{\|}$(начало отсчета совпадает с центром одной из гранул, например, $\lambda$ ). Площадь поперечного сечения межгранульной области интегрирования, отвечающая $\xi \approx 1 / 2$, равна $S_{m} \approx \pi r_{\perp}^{2} \approx \pi \gamma^{-1} R$. Соответственно, объем межгранульной области интегрирования равен $V_{b}=r_{\lambda, \lambda^{\prime}} \int_{R / 2 R+w}^{R+w / 2 R+w} S d \xi \approx S_{m} w ;$ т. е. можно считать, что межгранульная область представляет собой цилиндр с высотой $w$ и площадью основания $S_{m}$.

Вклад в интеграл неортогональности $s_{\lambda, \lambda^{\prime}}$ также вносит интегрирование по двум эффективным контактным областям (шаровым сегментам с высотой $\gamma^{-1}$ и площадью $S_{c} \approx S_{m} \approx \pi \gamma^{-1} R ; V_{c} \approx S_{c} \gamma^{-1}-$ объем эффективной контактной области), в области которых произведение $\psi_{\lambda} \psi_{\lambda^{\prime}}$ можно считать постоянным $\psi_{\lambda} \psi_{\lambda^{\prime}} \approx \psi_{\lambda}(R) \psi_{\lambda^{\prime}}(R+w)=\psi_{\lambda}(R+w) \psi_{\lambda^{\prime}}(R)$ $\approx(A \sin (\alpha R) / R)^{2} \exp \left(-\gamma w_{\lambda, \lambda^{\prime}}\right)$ и равным (9). 

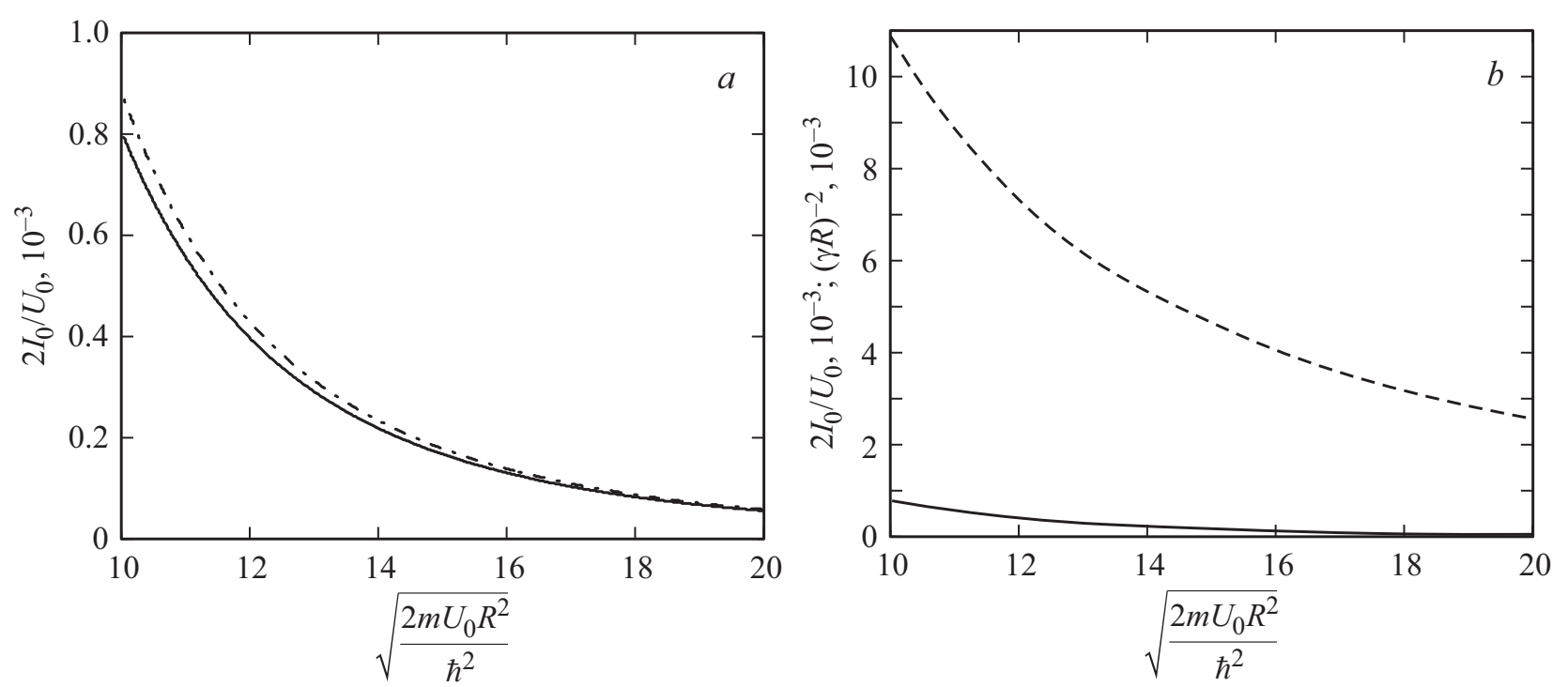

Рис. 1. $a-$ сплошная линия - график зависимости предэкспоненциального множителя $I_{0}$ резонансного интеграла $I_{\lambda, \lambda^{\prime}}(14)$ от безразмерного параметра $\sqrt{\frac{2 m U_{0} R^{2}}{\hbar^{2}}} ;$ здесь $I_{0} \approx \frac{U_{0}}{2} \frac{1}{\left(1-\frac{\sin (2 \alpha R)}{2 \alpha R}\right)}\left(\frac{\sin (\alpha R)}{\gamma R}\right)^{2}$. Для глубокой квантовой ямы предэкспоненциальный множитель $I_{0}$ становится равен $I_{0} \approx \frac{U_{0}}{2}\left(\frac{\sin (\alpha R)}{\gamma R}\right)^{2}$ (штрихпунктирная линия). $b-$ сплошная линия - график зависимости предэкспоненциального множителя $I_{0}$ резонансного интеграла $I_{\lambda, \lambda^{\prime}}(14)$ от безразмерного параметра $\sqrt{\frac{2 m U_{0} R^{2}}{\hbar^{2}}} ;$ где $I_{0} \approx \frac{U_{0}}{2} \frac{1}{\left(1-\frac{\sin (2 \alpha R)}{2 \alpha R}\right)}\left(\frac{\sin (\alpha R)}{\gamma R}\right)^{2}$. Для сравнения приведен график зависимости $(\gamma R)^{-2}$ от $\sqrt{\frac{2 m U_{0} R^{2}}{\hbar^{2}}}$ (пунктирная линия).

Таким образом, оценка интеграла неортогональности дает

$$
\begin{aligned}
s_{\lambda, \lambda^{\prime}} & =\left\langle\psi_{\lambda^{\prime}} \mid \psi_{\lambda}\right\rangle=\int_{V} \psi_{\lambda^{\prime}} \psi_{\lambda} d \mathbf{r} \approx \psi_{\lambda^{\prime}} \psi_{\lambda} \int_{V_{b}+2 V_{c}} d \mathbf{r} \\
& \approx\left(\frac{A \sin (\alpha R)}{R}\right)^{2} \exp \left(-\gamma w_{\lambda, \lambda^{\prime}}\right)\left(V_{b}+2 V_{c}\right)
\end{aligned}
$$

где $V_{b}+2 V_{c}=S_{m}\left(w+2 \gamma^{-1}\right)$ - объем цилиндра с площадью основания $S_{m}$ и высотой $w+2 \gamma^{-1}$, параллельной отрезку $r_{\lambda, \lambda^{\prime}}$.

В случае глубоких квантовых ям неравенство $\left|\left\langle\psi_{\lambda}\left|\hat{\tilde{U}}_{\lambda}\right| \psi_{\lambda^{\prime}}\right\rangle\right|>\left|e \varphi\left(\mathbf{r}_{\lambda}\right) s_{\lambda, \lambda^{\prime}}\right|$ выполняется и резонансный интеграл равен

$$
\begin{aligned}
I_{\lambda, \lambda^{\prime}} & =\left\langle\psi_{\lambda}\left|\hat{U}_{\lambda}\right| \psi_{\lambda^{\prime}}\right\rangle \approx\left\langle\psi_{\lambda}\left|\hat{\tilde{U}}_{\lambda}\right| \psi_{\lambda^{\prime}}\right\rangle \\
& =-U_{0} \int_{V_{\lambda}} \psi_{\lambda} \psi_{\lambda^{\prime}} d \mathbf{r} \approx-U_{0} \psi_{\lambda}(R) \psi_{\lambda^{\prime}}(R+w) V_{c}
\end{aligned}
$$

где $V_{\lambda}$ - объем наногранулы, отвечающей квантовой яме $\lambda$, по которому ведется интегрирование; с учетом (9) выражение для резонансного интеграла имеет вид

$$
I_{\lambda, \lambda^{\prime}} \approx-U_{0}\left(\frac{A \sin (\alpha R)}{R}\right)^{2} \exp \left(-\gamma w_{\lambda, \lambda^{\prime}}\right) V_{c}
$$

Оценки интеграла неортогональности (11) и резонансного интеграла (12.2) дают возможность представить неравенство (8) $\left|\left\langle\psi_{\lambda}\left|\tilde{\tilde{U}}_{\lambda}\right| \psi_{\lambda^{\prime}}\right\rangle\right|>\left|e \varphi\left(\mathbf{r}_{\lambda}\right) s_{\lambda, \lambda^{\prime}}\right|$ в виде условия на глубину квантовой ямы $U_{0} V_{c}>\left|e \varphi\left(\mathbf{r}_{\lambda}\right)\right|\left(V_{b}+2 V_{c}\right)$, т. е.

$$
U_{0}>\frac{e^{2}}{\kappa(d+w)} \frac{\left(w+2 \gamma^{-1}\right)}{\gamma^{-1}} \sim \frac{e^{2}}{\kappa d} \gamma w,
$$

где характерная энергия разброса уровней за счет случайных кулоновских полей порядка $\left|e \varphi\left(\mathbf{r}_{\lambda}\right)\right| \sim \frac{e^{2}}{\kappa(d+w)}$, $w$ - среднее расстояние между поверхностями ближайших гранул, $\gamma w \gg 1$.

C учетом выражений для коэффициента $A$ (5) и объема шарового сегмента (эффективной контактной области) $V_{c} \approx \pi \gamma^{-2} R$ резонансный интеграл (12.2) принимает вид

$$
I_{\lambda, \lambda^{\prime}} \approx-\frac{U_{0}}{2} \frac{1}{\left(1-\frac{\sin (2 \alpha R)}{2 \alpha R}\right)}\left(\frac{\sin (\alpha R)}{\gamma R}\right)^{2} \exp \left(-\gamma w_{\lambda, \lambda^{\prime}}\right) .
$$

Согласно (14), для сферических наногранул резонансный интеграл $I_{\lambda, \lambda^{\prime}}=I_{0} \exp \left(-w_{\lambda \lambda^{\prime}} \gamma\right)$ экспоненциально убывает с ростом расстояния $w_{\lambda \lambda^{\prime}}$ между поверхностями гранул $\lambda$ и $\lambda^{\prime}$.

Оценим предэкспоненциальный множитель $I_{0}$ резонансного интеграла. Из условия непрерывности логарифмической производной волновой функции на границе 
квантовой ямы следует, что $k_{2}=-k_{1} \operatorname{ctg}\left(k_{1}\right)$, где $k_{1}=$ $=\alpha R, \quad k_{2}=\gamma R$. Решение системы из двух трансцендентных уравнений $k_{2}=-k_{1} \operatorname{ctg}\left(k_{1}\right)$ и $k_{1}^{2}+k_{2}^{2}=$ $=2 m U_{0} R^{2} / \hbar^{2}\left(\alpha^{2}+\gamma^{2}=2 m U_{0} / \hbar^{2}\right)$, дает значения энергий, отвечающих связанным состояниям в сферической прямоугольной квантовой яме (см., например, [24]). Из решения следует, что в случае глубокой квантовой ямы $2 m U_{0} R^{2} / \hbar^{2} \gg \pi^{2}$ для основного состояния имеем $k_{1}=\alpha R \rightarrow \pi, k_{2}=\gamma R \rightarrow \sqrt{2 m U_{0} R^{2} / \hbar^{2}}$; при этом резонансный интеграл (14) принимает вид (рис. 1,a)

$$
\begin{aligned}
I_{\lambda, \lambda^{\prime}} & \approx-\frac{U_{0}}{2}\left(\frac{\sin (\alpha R)}{\gamma R}\right)^{2} \exp \left(-\gamma w_{\lambda, \lambda^{\prime}}\right) \\
& =-\frac{1}{4} \frac{\hbar^{2}}{m R^{2}}(\sin (\alpha R))^{2} \exp \left(-\gamma w_{\lambda, \lambda^{\prime}}\right) .
\end{aligned}
$$

Соответственно, в случае сферических наногранул предэкспоненциальный множитель

$$
I_{0}=\frac{U_{0}}{2}\left(\frac{\sin (\alpha R)}{\gamma R}\right)^{2}=\frac{1}{4} \frac{\hbar^{2}}{m R^{2}}(\sin (\alpha R))^{2}
$$

резонансного интеграла $I_{\lambda, \lambda}(15)$ существенно уменьшается с увеличением линейного размера гранул (рис. 1, a); при этом фактор ослабления предэкспоненциального множителя $I_{0}$ равен $(\sin (\alpha R) / \gamma R)^{2}$ (рис. $\left.1, b\right)$.

\section{4. Частотная зависимость бесфононной проводимости}

Переход от суммирования к интегрированию в выражении для проводимости (4) можно осуществить через функцию распределения $P\left(w_{\lambda \lambda^{\prime}}\right)$ величин расстояний между поверхностями гранул [25]

$$
\begin{aligned}
& \sum_{\lambda, \lambda^{\prime}} \ldots \rightarrow \frac{V_{0}}{d^{3}} g_{0}^{2} \int_{B}^{A} d \varepsilon_{\lambda}^{0} \int_{\varepsilon_{\lambda}^{0}}^{A} d \varepsilon_{\lambda^{\prime}}^{0} \int P\left(w_{\lambda \lambda^{\prime}}\right) d w_{\lambda \lambda^{\prime}} d \widetilde{\Omega} \ldots, \\
& P\left(w_{\lambda \lambda^{\prime}}\right)=\frac{p\left(w_{\lambda \lambda^{\prime}}\right)}{\int p\left(w_{\lambda \lambda^{\prime}}\right) d w_{\lambda \lambda^{\prime}} d \widetilde{\Omega}}=\frac{1}{4 \pi} \frac{p\left(w_{\lambda \lambda^{\prime}}\right)}{p\left(w_{\lambda \lambda^{\prime}}\right) d w_{\lambda \lambda^{\prime}}},
\end{aligned}
$$

где $g_{0}$ - одночастичная плотность состояний в расчете на центр, принимаемая равной постоянной величине, $p\left(w_{\lambda \lambda^{\prime}}\right)$ - плотность вероятности того, что расстояние между поверхностями соседних гранул равно $w_{\lambda \lambda^{\prime}}, \widetilde{\Omega}-$ сферический угол. Одночастичную плотность состояний в расчете на центр следует рассматривать, как плотность вероятности того, что энергия основного состояния в грануле равна $\varepsilon_{\lambda}^{0}$.

Как и в теории бесфононной проводимости для систем с точечными центрами локализации, учет кулоновского взаимодействия между электронами, локализованными на рассматриваемой паре центров (гранул), вместе с законом сохранения энергии $\varepsilon_{\lambda, \lambda^{\prime}}^{+}=\varepsilon_{\lambda, \lambda^{\prime}}^{-}+\hbar \omega$ при низких температурах приводят к тому, что разность средних чисел заполнения отлична от нуля и равна единице $n_{F}\left(\varepsilon_{\lambda, \lambda^{\prime}}^{-}\right)-n_{F}\left(\varepsilon_{\lambda, \lambda^{\prime}}^{+}\right)=1$ при $\mu-\hbar \omega-\frac{e^{2}}{\kappa r_{\lambda, \lambda^{\prime}}}<\varepsilon_{\lambda, \lambda^{\prime}}^{-}<\mu$.

С учетом (17) для вещественной части бесфононной проводимости получаем

$$
\begin{aligned}
\sigma_{1}^{\mathrm{res}}(\omega)= & \frac{4 \pi^{2} e^{2} g_{0}^{2}}{3 \hbar d^{3}} \int_{w_{\omega}}^{\infty}\left(\hbar \omega+\frac{e^{2}}{\kappa\left(d+w_{\lambda \lambda^{\prime}}\right)}\right) \\
& \times \frac{\left(d+w_{\lambda \lambda^{\prime}}\right)^{2} I_{\lambda \lambda^{\prime}}^{2} P\left(w_{\lambda \lambda^{\prime}}\right)}{\sqrt{(\hbar \omega)^{2}-4 I_{\lambda \lambda^{\prime}}^{2}}} d w_{\lambda \lambda^{\prime}},
\end{aligned}
$$

где $\quad 2 I_{\lambda \lambda^{\prime}}\left(w_{\omega}\right)=\hbar \omega, \quad w_{\omega}=\gamma^{-1} \ln \left(2 I_{0} / \hbar \omega\right), \quad I_{\lambda \lambda^{\prime}}=$ $=I_{0} \exp \left(-w_{\lambda \lambda^{\prime}} \gamma\right), d-$ средний диаметр гранулы, определяющий изменение дипольного момента системы при электронных переходах, $\kappa-$ диэлектрическая проницаемость среды. Из-за гибридизации волновых функций изолированной пары центров и соответствующего ей отталкивания уровней, наибольший вклад в бесфононную проводимость при частотах $\omega<\omega_{c}$ вносят пары центров, для которых расстояние между поверхностями $w_{\lambda \lambda^{\prime}}$ (длина туннелирования) удовлетворяет неравенствам $w_{\omega}<w_{\lambda \lambda^{\prime}}<w_{\omega}+\gamma^{-1}$; при $w_{\lambda \lambda^{\prime}}<w_{\omega}$ отталкивание уровней становится большим $\hbar \omega$, так что переходы невозможны, а с увеличением межцентрового расстояния в паре экспоненциально уменьшается перекрытие волновых функций состояний. Нижний предел интегрирования в выражении (18) равен $w_{\omega}$ при условии $\omega<\omega_{c}$ и нулю в случае $\omega \geq \omega_{c}$, где критическая частота $\omega_{c}=2 I_{0} / \hbar$. При частотах $\omega \geq \omega_{c}$ длина туннелирования $w_{\omega}$ становится порядка длины затухания $\gamma^{-1}$ волновой функции в области барьера; формально при $\omega=\omega_{c}$ длина $w_{\omega}$ обращается в нуль.

Отметим, что проводимость определяется переходами между ближайшими соседями (гранулами) в области высоких частот, для которых $w_{\omega}<d$ (т. е. $\omega>\omega_{c} \exp (-\gamma d)$, где $\left.\gamma d \gg 1\right)$. В низкочастотном диапазоне, отвечающем $w_{\omega}>d$, проводимость определяется переходами между удаленными (не ближайшими) соседями через промежуточные виртуальные состояния, которые обусловлены гибридизацией состояний группы соседних гранул [20-22]. С ростом частоты переход к режиму проводимости между ближайшими соседями происходит при частотах, отвечающих $w_{\omega} \sim d$. Вследствие уменьшения предэкспоненциального множителя резонансного интеграла $I_{0}$ для гранулированных систем переход к режиму проводимости с постоянной длиной прыжка происходит при существенно меньших частотах, чем в случае систем с водородоподобными центрами локализации.

Согласно (18), в рассматриваемом случае плотной упаковки гранул, выражение для вещественной части низкотемпературной бесфононной проводимости, вычисленной в парном приближении с учетом как квантового отталкивания уровней, так и кулоновского взаимодей- 
ствия между электронами, принимает вид

$$
\begin{aligned}
\sigma_{1}^{\mathrm{res}}(\omega)= & \frac{4 \pi^{2} e^{2} g_{0}^{2}}{3 \hbar d}(\hbar \omega+U) \\
& \times \int_{w_{\omega}}^{\infty} P\left(w_{\lambda \lambda^{\prime}}\right) \frac{I_{\lambda \lambda^{\prime}}^{2}}{\sqrt{(\hbar \omega)^{2}-4 I_{\lambda \lambda^{\prime}}^{2}}} d w_{\lambda \lambda^{\prime}},
\end{aligned}
$$

где $U \approx e^{2} / \kappa d-$ характерная энергия кулоновского взаимодействия электронов внутри пар. Выражение (19) дает обобщение теории резонансной прыжковой проводимости, учитывающей квантовую корреляцию состояний и кулоновское взаимодействие, на случай систем с центрами локализации конечных размеров. Отметим, что полученное выражение (19) по структуре сходно с выражением для резонансной проводимости систем с точечными центрами локализации. В (19) кроме множителя $\hbar \omega+U$ (где $U$ слабо зависит от частоты) есть и зависящий от частоты интеграл, по виду сходный с интегралом $\int_{r_{\omega}}^{\infty} \frac{I_{\lambda \lambda^{\prime}}^{2}}{\sqrt{(\hbar \omega)^{2}-4 I_{\lambda \lambda^{\prime}}^{2}}} d r_{\lambda \lambda^{\prime}}=\frac{a \hbar \omega}{4}$ в теории для точечных центров локализации; $r_{\omega}=a \ln \left(\omega_{c} / \omega\right)-$ характерная длина прыжка на частоте $\omega, a-$ радиус локализации состояний, $\omega_{c}=2 I_{0} / \hbar-$ критическая частота, $I_{0}$ - предэкспоненциальный множитель резонансного интеграла $I_{\lambda \lambda^{\prime}}=I_{0} \exp \left(-r_{\lambda \lambda^{\prime}} / a\right)$, а $r_{\lambda \lambda^{\prime}}-$ расстояние между центрами $\lambda, \lambda^{\prime}$, (межцентровое расстояние в паре) $[7,8]$.

\section{5. Особенности поведения частотной зависимости бесфононной проводимости}

Из обобщения теории резонансной прыжковой проводимости неупорядоченных систем с точечными центрами на системы с областями локализации конечных размеров (массивы наногранул или квантовых точек) следует, что в области промежуточных частот, $d>w_{\omega}>\gamma^{-1}$, при условии слабой (неэкспоненциальной) зависимости $P(w)$ частотная зависимость бесфононной проводимости имеет универсальной степенной вид $\sigma_{1}^{\text {res }}(\omega) \sim \omega^{s}$, где $s \approx 1$.

Действительно, в области промежуточных частот, считая, что ширина распределения $P(w)$ велика по сравнению с $\gamma^{-1}$, вынесем функцию $P\left(w_{\lambda \lambda^{\prime}}\right)$ в (19) за знак интеграла в точке $w_{\lambda \lambda^{\prime}}=w_{\omega}$; здесь $P(w)-$ медленно меняющаяся функция в окрестности $w_{\omega}$. С учетом равенства $\int_{w_{\omega}}^{\infty} \frac{I_{\lambda \lambda^{\prime}}^{2}}{\sqrt{(\hbar \omega)^{2}-4 I_{\lambda \lambda^{\prime}}^{2}}} d w_{\lambda \lambda^{\prime}}=\frac{\gamma^{-1} \hbar \omega}{4}$ получаем

$$
\sigma_{1}^{\mathrm{res}}(\omega)=\frac{\pi^{2} e^{2} \gamma^{-1} g_{0}^{2}}{3 d}\left(\hbar \omega+\frac{e^{2}}{\kappa d}\right) \omega P\left(w_{\omega}\right) .
$$

Согласно (20), характерная частотная зависимость и величина проводимости зависят от размеров областей локализации (средний диаметр гранул $d=2 R$ ). Для характерных значений $I_{0} / U_{0} \sim 10^{-4}, \sqrt{2 m U_{0} R^{2} / \hbar^{2}} \sim 10$, $m=0.1 m_{0} \quad\left(m_{0}-\right.$ масса свободного электрона), $U_{0} \sim 300 \mathrm{meV}$ оценка критической частоты и радиуса наногранулы дает $v_{c}=2 I_{0} / h \sim 10 \mathrm{GHz}\left(I_{0} \sim 0.03 \mathrm{meV}\right)$ и $R \approx 100 \AA$; при этом частота перехода от линейной к квадратичной частотной зависимости проводимости, определяемая равенством $\hbar \omega_{\mathrm{cr}}=e^{2} / \kappa d$, порядка $\quad v_{\mathrm{cr}}=\omega_{\mathrm{cr}} / 2 \pi \sim 1 \mathrm{THz} \quad$ (при $\quad \kappa=10 \quad$ имеем $\left.e^{2} / \kappa d \approx 4 \mathrm{meV}\right)$. Соответственно, вплоть до критической частоты $\omega_{c}=2 \pi v_{c}=2 I_{0} / \hbar$ кулоновское взаимодействие между электронами на резонансной паре центров локализации играет определяющую роль, $e^{2} / \kappa d>\hbar \omega_{c}$, и частотная зависимость бесфононной проводимости принимает вид

$$
\sigma_{1}^{\mathrm{res}}(\omega)=\frac{\pi^{2} e^{4} \gamma^{-1} g_{0}^{2}}{3 \kappa d^{2}} \omega P\left(w_{\omega}\right)
$$

здесь $\omega_{c}>\omega>\omega_{c} \exp (-\gamma d)\left(\right.$ т. е. $\left.\gamma^{-1}<w_{\omega}<d\right)$.

Согласно (21), в промежуточной области частот, $d>w_{\omega}>\gamma^{-1}$, на проводимость влияет функция распределения $P(w)$, что может приводить к отклонениям от линейной частотной зависимости проводимости (универсальности). Отметим, что учет размеров гранул в случае высокочастотной релаксационной проводимости также дает частотную зависимость вида $\sigma_{1}^{\text {rel }}(\omega) \sim \omega P\left(\widetilde{w}_{\omega}\right)$ (здесь $\tilde{w}_{\omega}=(2 \gamma)^{-1} \ln \left(\omega_{\mathrm{ph}} / \omega\right), \omega_{\mathrm{ph}}-$ характерная фононная частота, $\omega_{\text {ph }} \sim 10^{12}-10^{13} \mathrm{rad} / \mathrm{sec}$ ) [20].

Поскольку средний диаметр гранул $d$ существенно превышает расстояния между поверхностями соседних гранул $w_{\lambda \lambda^{\prime}}$, изменение дипольного момента системы при электронных переходах в высокочастотной области не зависит от частоты и определяется средним диаметром гранулы; при этом в выражении для проводимости массива наногранул не фигурируют логарифмические множители вида $\ln \left(\omega_{\mathrm{c}, \mathrm{ph}} / \omega\right)$, связанные с частотной зависимостью длины прыжка $r_{\omega}$.

В окрестности частоты $\omega \sim \omega_{c}$ выражение (19) предсказывает замедление роста вещественной части проводимости с частотой и асимптотический выход на насыщение через область уменьшения $\sigma_{1}(\omega)$ при $\omega>\omega_{c}$.

В области частот $e^{2} / \kappa d>\hbar \omega>\hbar \omega_{c}$ частотная зависимость проводимости (19) принимает вид

$$
\begin{aligned}
\sigma_{1}^{\mathrm{res}} & =\frac{4 \pi^{2} e^{2} g_{0}^{2}}{3 \hbar d} \frac{e^{2}}{\kappa d \hbar \omega} \int_{0}^{\infty} P\left(w_{\lambda \lambda^{\prime}}\right) I_{\lambda \lambda^{\prime}}^{2} d w_{\lambda \lambda^{\prime}} \\
& \approx \frac{2 \pi^{2} e^{2} g_{0}^{2} \gamma^{-1}}{3 \hbar d} \frac{e^{2}}{\kappa d \hbar \omega} P\left(\gamma^{-1}\right) I_{0}^{2},
\end{aligned}
$$

где $\int_{0}^{\infty} d w P(w) I^{2}(w) \approx(2 \gamma)^{-1} P\left(\gamma^{-1}\right) I_{0}^{2}$. Согласно (22), в области промежуточных частот аппроксимация частотной зависимости проводимости степенной функцией приводит к отрицательному значению показателя 


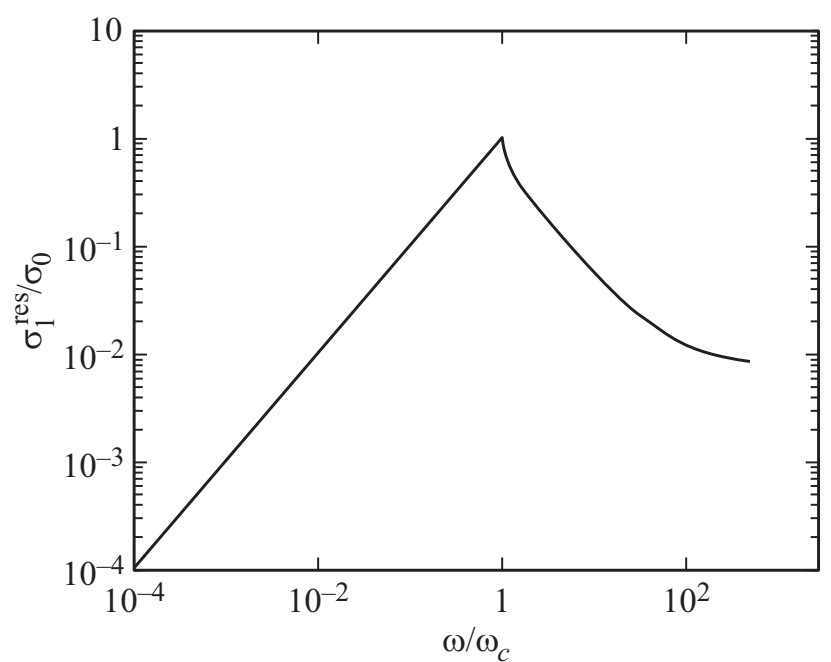

Рис. 2. Частотная зависимость вещественной части бесфононной проводимости массива наногранул, вычисленная на основе выражения (19) при условии $P(w)=$ const; здесь $\sigma_{0}=\frac{\pi^{2} e^{4} \gamma^{-1} g_{0}^{2}}{3 \kappa d^{2}} \omega_{c} P$.

степени $s$. Немонотонность $\sigma_{1}^{\text {res }}(\omega)$ с ростом частоты при $\omega \sim \omega_{c}$ обусловлена тем, что при более низких частотах $\omega<\omega_{c}$ пары центров, для которых расстояние между поверхностями $w_{\lambda \lambda^{\prime}}<w_{\omega}$, из-за отталкивания уровней, вклада в проводимость давать не могут; в то время как при более высоких частотах $\omega>\omega_{c}$ такого пространственного ограничения на расстояние между поверхностями гранул $w_{\lambda \lambda^{\prime}}$ нет $\left(\hbar \omega>2 I_{\lambda \lambda^{\prime}}\right)$.

Из выражения (19) можно оценить величину бесфононной проводимости в области насыщения, $\hbar \omega>e^{2} / \kappa d$,

$$
\begin{aligned}
\sigma_{1, \mathrm{sat}}^{\mathrm{res}} & =\frac{4 \pi^{2} e^{2} g_{0}^{2}}{3 \hbar d} \int_{0}^{\infty} P\left(w_{\lambda \lambda^{\prime}}\right) I_{\lambda \lambda^{\prime}}^{2} d w_{\lambda \lambda^{\prime}} \\
& \approx \frac{2 \pi^{2} e^{2} g_{0}^{2} \gamma^{-1}}{3 \hbar d} P\left(\gamma^{-1}\right) I_{0}^{2}
\end{aligned}
$$

при этом выражение (23) можно представить в виде $\sigma_{1, \mathrm{sat}}^{\mathrm{res}} \approx \frac{\pi^{2} e^{2} g_{0}^{2} \gamma^{-1}}{3 d} P\left(\gamma^{-1}\right) \omega_{c} I_{0}$.

Согласно (21), бесфононная проводимость в окрестности критической частоты $\omega \sim \omega_{c}$, равна $\sigma_{1}^{\mathrm{res}}\left(\omega_{c}\right)=\frac{\pi^{2} e^{4} \gamma^{-1} g_{0}^{2}}{3 \kappa d^{2}} \omega_{c} P\left(w_{\omega} \approx \gamma^{-1}\right)$, то есть $\frac{\sigma_{1}^{\mathrm{res}}\left(\omega_{c}\right)}{\sigma_{1, \mathrm{sat}}^{\mathrm{res}}} \approx$ $\approx \frac{e^{2}}{\kappa d I_{0}}$; при этом $\sigma_{1}^{\text {res }}\left(\omega_{c}\right)>\sigma_{1, \text { sat }}^{\text {res }}$. Действительно, для характерных значений $I_{0} / U_{0} \sim 10^{-4}, \quad U_{0} \sim 300 \mathrm{meV}$ предэкспоненциальный множитель резонансного интеграла порядка $I_{0} \sim 0.03 \mathrm{meV}\left(v_{c}=2 I_{0} / h \sim 10 \mathrm{GHz}\right)$; значение радиуса наногранулы $R \approx 100 \AA$ отвечает $e^{2} / \kappa d \approx 4 \mathrm{meV}$, т. е. $\sigma_{1}^{\text {res }}\left(\omega_{c}\right) / \sigma_{1 \text { sat }}^{\text {res }} \approx e^{2} / \kappa d I_{0} \sim 10^{2}$. Следовательно, в области частот $\omega \sim \omega_{c}$ можно ожидать отклонений от универсальности, связанных с ослаблением частотной зависимости проводимости и ее немонотонности; при этом для характерных значений параметров при $v>e^{2} / h \kappa d \sim 1 \mathrm{THz}$ можно ожидать насыщения проводимости. На рис. 2 представлена частотная зависимость бесфононной проводимости на переменном токе, вычисленная на основе выражения (19) при $P(w)=$ const (случай малых энергетических потерь $s \approx 1$ ). Важной особенностью выражения (19) является немонотонность частотной зависимости проводимости; в области частот $\omega>\omega_{c}$ проводимость уменьшается с ростом частоты.

Немонотонность частотной зависимости проводимости $\sigma_{1}(\omega)$ гранулированной системы в окрестности частоты $\omega \sim \omega_{c}$ может быть связана с подавлением гибридизации электронных состояний; при этом с ростом частоты основной вклад в проводимость начинают вносить пары центров, в которых электрон не успевает гибридизоваться. Для заданной пары центров $\lambda, \lambda^{\prime}$ гибридизация характеризуется частотой Раби $\omega_{\mathrm{R}}=2 I\left(w_{\lambda \lambda^{\prime}}\right) / \hbar$. Согласно (18), на частоте $\omega<\omega_{c}$ основной вклад в проводимость дают пары с межгранульными расстояниями $w_{\omega}<w_{\lambda \lambda^{\prime}}<w_{\omega}+\gamma^{-1}$. Вместе с тем для пар с межцентровыми расстояниями $w_{\lambda \lambda^{\prime}}>w_{\omega}$ имеем $\omega>\omega_{\mathrm{R}}$; при этом эффекты, связанные с гибридизацией локализованных состояний, становятся несущественны. В этом случае матричный элемент в выражении для проводимости

$$
\begin{aligned}
& \sigma_{1}^{\mathrm{res}}(\omega)=\frac{\pi e^{2} \omega}{V_{0}} \sum_{\substack{\left\{\lambda \lambda^{\prime}\right\} \\
\lambda \neq \lambda^{\prime}}}\left|\left\langle\psi_{\lambda^{\prime}}|(\mathbf{n}, \mathbf{r})| \psi_{\lambda}\right\rangle\right|^{2} \\
& \quad \times\left(n_{F}\left(\varepsilon_{\lambda^{\prime}}\right)-n_{F}\left(\varepsilon_{\lambda}\right)\right) \delta\left(\varepsilon_{\lambda}-\varepsilon_{\lambda^{\prime}}-\frac{e^{2}}{\kappa r_{\lambda^{\prime} \lambda}}-\hbar \omega\right)
\end{aligned}
$$

равен

$$
\begin{aligned}
\left\langle\psi_{\lambda^{\prime}}|(\mathbf{n}, \mathbf{r})| \psi_{\lambda}\right\rangle & \approx \int_{V_{b}+2 V_{c}} \psi_{\lambda^{\prime}} \psi_{\lambda} r_{\|} \cos \theta d \mathbf{r} \\
& \left.\approx \psi_{\lambda^{\prime}} \psi_{\lambda}\right|_{V_{b}+2 V_{c}}\left(V_{b}+2 V_{c}\right) R \cos \theta
\end{aligned}
$$

где $d \mathbf{r}=S d r_{\|}, \Delta H_{\lambda, \lambda^{\prime}}=\varepsilon_{\lambda}-\varepsilon_{\lambda^{\prime}}-\frac{e^{2}}{\kappa r_{\lambda^{\prime}, \lambda}}-$ изменение энергии при одноэлектронном переходе из состояния $\lambda^{\prime}$ в состояние $\lambda, \varepsilon_{\lambda}, \varepsilon_{\lambda^{\prime}}-$ самосогласованные энергии электрона, отвечающие состояниям $\lambda$ и $\lambda^{\prime}, \theta-$ угол между векторами $\mathbf{n}$ и $\mathbf{r}_{\lambda \lambda^{\prime}}$; с учетом (9) имеем

$$
\begin{aligned}
\left\langle\psi_{\lambda^{\prime}}|(\mathbf{n}, \mathbf{r})| \psi_{\lambda}\right\rangle & \approx\left(\frac{A \sin (\alpha R)}{R}\right)^{2} \exp \left(-\gamma w_{\lambda, \lambda^{\prime}}\right) \\
& \times S_{m}\left(w_{\lambda, \lambda^{\prime}}+2 \gamma^{-1}\right) R \cos \theta .
\end{aligned}
$$

При низких температурах в качестве начального состояния можно выбрать основное состояние системы; тогда все состояния с энергиями $\varepsilon_{\lambda^{\prime}}<\mu$ заняты, а с энергиями $\varepsilon_{\lambda}>\mu$ свободны. Разность чисел заполнения отлична от нуля и равна единице, $n_{F}\left(\varepsilon_{\lambda^{\prime}}\right)-n_{F}\left(\varepsilon_{\lambda}\right)=1$, при условии $\varepsilon_{\lambda^{\prime}}<\mu, \varepsilon_{\lambda}=\varepsilon_{\lambda^{\prime}}+\frac{e^{2}}{\kappa r_{\lambda^{\prime}, \lambda}}+\hbar \omega>\mu$, т. е. при выполнении неравенств $\mu-\hbar \omega-\frac{e^{2}}{\kappa r_{\lambda^{\prime}, \lambda}}<\varepsilon_{\lambda^{\prime}}<\mu$. 
С учетом (17), для резонансной проводимости имеем

$$
\begin{aligned}
& \sigma_{1}^{\mathrm{res}}(\omega)=\frac{\pi e^{2} g_{0}^{2} \omega}{d^{3}} \int_{-\left(\hbar \omega+\frac{e^{2}}{\kappa d}\right)}^{0} d \varepsilon_{\lambda^{\prime}} \int d \varepsilon_{\lambda} \int d \widetilde{\Omega} d w_{\lambda \lambda^{\prime}} \\
& \times P\left(w_{\lambda^{\prime} \lambda}\right)\left|\left\langle\psi_{\lambda^{\prime}}|(\mathbf{n}, \mathbf{r})| \psi_{\lambda}\right\rangle\right|^{2} \delta\left(\varepsilon_{\lambda}-\varepsilon_{\lambda^{\prime}}-\frac{e^{2}}{\kappa r_{\lambda^{\prime} \lambda}}-\hbar \omega\right),
\end{aligned}
$$

где $\int(\cos \theta)^{2} d \widetilde{\Omega}=4 \pi / 3$; энергия отсчитывается от уровня Ферми $\mu=0$. Плотность состояний в (26) можно записать в факторизованном виде, поскольку вклад в бесфононную проводимость вносят переходы вне кулоновской щели; при этом плотность состояний близка к затравочной $g_{0}$. Интегрируя (26), с учетом (25.2) получаем

$$
\begin{aligned}
\sigma_{1}^{\mathrm{res}}(\omega)= & \frac{\pi^{2} e^{2} g_{0}^{2} \gamma^{2}}{3 d}\left(\frac{I_{0}}{U_{0}}\right)^{2}\left(\hbar \omega+\frac{e^{2}}{\kappa d}\right) \omega \\
& \times \int_{0}^{\infty} P(w)\left(w+2 \gamma^{-1}\right)^{2} \exp (-2 \gamma w) d w
\end{aligned}
$$

где $\quad \int_{0}^{\infty} P(w)\left(w+2 \gamma^{-1}\right)^{2} \exp (-2 \gamma w) d w \approx P\left(\gamma^{-1}\right) / 4 \gamma^{3}$; при этом выражение для вещественной части бесфононной проводимости принимает вид

$$
\sigma_{1}^{\mathrm{res}}(\omega)=\frac{\pi^{2} e^{2} \gamma^{-1} g_{0}^{2}}{3 d}\left(\frac{I_{0}}{2 U_{0}}\right)^{2}\left(\hbar \omega+\frac{e^{2}}{\kappa d}\right) \omega P\left(\gamma^{-1}\right)
$$

Таким образом, с учетом подавления гибридизации состояний в области частот $\omega>\omega_{c}$ фактор уменьшения проводимости равен $I_{0}^{2} / U_{0}^{2} \sim 10^{-7}$ (рис. $\left.1, a, b\right)$.

\section{6. Заключение}

Обобщение теории бесфононной проводимости систем с точечными примесными центрами на системы с областями локализации конечных размеров (массивы наногранул или квантовых точек) показало, что в промежуточной области частот, $d>w_{\omega}>\gamma^{-1}$, проводимость зависит от функции распределения величин расстояний между поверхностями гранул $P(w)$; это может приводить к отклонениям от линейной частотной зависимости вещественной части проводимости. В окрестности частоты $\omega \sim \omega_{c}$ для неупорядоченных гранулированных систем следует ожидать отклонений от универсальности $(s \approx 1)$ частотной зависимости проводимости $\sigma_{1}(\omega)$ и ее немонотонности; при этом с увеличением размера гранул немонотонность $\sigma_{1}(\omega)$ должна проявляться при меньших частотах. Такое поведение частотной характеристики обусловлено тем, что для случая гранулированных систем частота $\omega_{c}$ заметно уменьшается по сравнению со значениями, характерными для систем точечных (водородоподобных) центров локализации, в связи с уменьшением предэкспоненциального множителя резонансного интеграла $I_{0}$.

\section{Список литературы}

[1] F. Kremer, A. Schonhals. Broadband dielectric spectroscopy. Springer, Berlin, N.Y. (2003). 729 p.

[2] I.P. Zvyagin. In: Charge Transport in Disordered Solids with Applications in Electronics / Ed. S. Baranovski. John Wiley \& Sons, Chichester (2006). P. 339.

[3] И.П. Звягин. Кинетические явления в неупорядоченных полупроводниках. Изд-во МГУ, М. (1984). 192 с.

[4] M. Pollak, T.H. Geballe. Phys. Rev. 122, 1742 (1961).

[5] I.G. Austin, N.F. Mott. Adv. Phys. 18, 41 (1969).

[6] A.L. Efros. Philos. Mag. B 43, 829 (1981).

[7] Б.И. Шкловский, А.Л. Эфрос. ЖЭТФ 81, 406 (1981).

[8] N.F. Mott. Philos. Mag. 22, 7 (1970).

[9] M. Lee, M.L. Stutzmann. Phys. Rev. Lett. 87, 056402 (2001).

[10] E. Helgren, N.P. Armitage, G. Gruner. Phys. Rev. B 69, 014201 (2004).

[11] M. Hering, M. Scheffler, M. Dressel, H. Lohneysen. Phys. Rev. B 75, 205203 (2007).

[12] J.A. Reedijk, L.J. Adriaanse, H.B. Brom, L.J. de Jongh, G. Schmid. Phys. Rev. B 57, R15116 (1998).

[13] И.П. Звягин, М.А. Ормонт. Вестн. МГУ. Физика, астрономия, 4, 44 (2008).

[14] М.А. Ормонт, И.П. Звягин. ФТП 49, 4, 449 (2015).

[15] М.А. Ормонт. ФТП 49, 10, 1314 (2015).

[16] М.А. Ормонт, И.П. Звягин. ФТП 52, 2, 161 (2018).

[17] М.А. Ормонт, И.П. Звягин. ФТТ, 60, 5, 880 (2018).

[18] A. Chandra Bose, P. Balaya, P. Thangadurai, S. Ramasamy. J. Phys. Chem. Solids 64, 659 (2003).

[19] H.B. Brom, M.A.J. Michels. Phil. Mag. B 81, 941 (2001).

[20] I.P. Zvyagin. In: Abstracts to 14 Int. Symp. „Nanostructures: Physics and Technology“, St.Petersburg, Russia, 2006. P. 226.

[21] I.P. Zvyagin. Phys. Stat. Solidi C 3, 300 (2006).

[22] I.P. Zvyagin, R. Keiper. Phil. Mag. B 81, 997 (2001).

[23] В.Л. Бонч-Бруевич, И.П. Звягин, Р. Кайпер, А.Г. Миронов, Р. Эндерлайн, Б. Эссер. Электронная теория неупорядоченных полупроводников. Наука, М. (1981). 384 с.

[24] А.С. Давыдов. Квантовая механика, Изд-во физ.-мат. лит., M. (1963).

[25] I.P. Zvyagin, M.A. Ormont. In: Reviews and Short Notes to Int. Conf. „Nanomeeting-2007“ / Eds V.E. Borisenko, S.V. Gaponenko, V.S. Gurin. Minsk (2007). P. 85.

Редактор Т.Н. Василевская 